\title{
A Convexity Property for an Integral Operator on the Class $S_{P}(\beta)$
}

\author{
Daniel Breaz \\ Department of Mathematics, "1 Decembrie 1918" University, Alba Iulia 510009, Romania
}

Correspondence should be addressed to Daniel Breaz, dbreaz@uab.ro

Received 30 October 2007; Accepted 30 December 2007

Recommended by Narendra Kumar K. Govil

We consider an integral operator, $F_{n}(z)$, for analytic functions, $f_{i}(z)$, in the open unit disk, $U$. The object of this paper is to prove the convexity properties for the integral operator $F_{n}(z)$, on the class $S_{p}(\beta)$.

Copyright (C) 2008 Daniel Breaz. This is an open access article distributed under the Creative Commons Attribution License, which permits unrestricted use, distribution, and reproduction in any medium, provided the original work is properly cited.

\section{Introduction}

Let $U=\{z \in C,|z|<1\}$ be the unit disc of the complex plane and denote by $H(U)$ the class of the holomorphic functions in $U$. Let $A=\left\{f \in H(U), f(z)=z+a_{2} z^{2}+a_{3} z^{3}+\cdots, z \in U\right\}$ be the class of analytic functions in $U$ and $S=\{f \in A: f$ is univalent in $U\}$.

Denote with $K$ the class of convex functions in $U$, defined by

$$
K=\left\{f \in A: \operatorname{Re}\left\{\frac{z f^{\prime \prime}(z)}{f^{\prime}(z)}+1\right\}>0, z \in U\right\} .
$$

A function $f \in S$ is the convex function of order $\alpha, 0 \leq \alpha<1$, and denote this class by $K(\alpha)$ if $f$ verifies the inequality

$$
\operatorname{Re}\left\{\frac{z f^{\prime \prime}(z)}{f^{\prime}(z)}+1>\alpha, z \in U\right\} .
$$

Consider the class $S_{p}(\beta)$, which was introduced by Ronning [1] and which is defined by

$$
f \in S_{p}(\beta) \Longleftrightarrow\left|\frac{z f^{\prime}(z)}{f(z)}-1\right| \leq \operatorname{Re}\left\{\frac{z f^{\prime}(z)}{f(z)}-\beta\right\}
$$

where $\beta$ is a real number with the property $-1 \leq \beta<1$. 
For $f_{i}(z) \in A$ and $\alpha_{i}>0, i \in\{1, \ldots, n\}$, we define the integral operator $F_{n}(z)$ given by

$$
F_{n}(z)=\int_{0}^{z}\left(\frac{f_{1}(t)}{t}\right)^{\alpha_{1}} \cdots\left(\frac{f_{n}(t)}{t}\right)^{\alpha_{n}} d t
$$

This integral operator was first defined by B. Breaz and N. Breaz [2]. It is easy to see that $F_{n}(z) \in A$.

\section{Main results}

Theorem 2.1. Let $\alpha_{i}>0$, for $i \in\{1, \ldots, n\}$, let $\beta_{i}$ be real numbers with the property $-1 \leq \beta_{i}<1$, and let $f_{i} \in S_{p}\left(\beta_{i}\right)$ for $i \in\{1, \ldots, n\}$.

If

$$
0<\sum_{i=1}^{n} \alpha_{i}\left(1-\beta_{i}\right) \leq 1
$$

then the function $F_{n}$ given by (1.4) is convex of order $1+\sum_{i=1}^{n} \alpha_{i}\left(\beta_{i}-1\right)$.

Proof. We calculate for $F_{n}$ the derivatives of first and second orders.

From (1.4) we obtain

$$
\begin{aligned}
& F_{n}^{\prime}(z)=\left(\frac{f_{1}(z)}{z}\right)^{\alpha_{1}} \cdots\left(\frac{f_{n}(z)}{z}\right)^{\alpha_{n}} \\
& F_{n}^{\prime \prime}(z)=\sum_{i=1}^{n} \alpha_{i}\left(\frac{f_{i}(z)}{z}\right)^{\alpha_{i}}\left(\frac{z f_{i}^{\prime}(z)-f_{i}(z)}{z f_{i}(z)}\right) \prod_{\substack{j=1 \\
j \neq i}}^{n}\left(\frac{f_{j}(z)}{z}\right)^{\alpha_{j}} .
\end{aligned}
$$

After some calculus, we obtain that

$$
\frac{F_{n}^{\prime \prime}(z)}{F_{n}^{\prime}(z)}=\alpha_{1}\left(\frac{z f_{1}^{\prime}(z)-f_{1}(z)}{z f_{1}(z)}\right)+\cdots+\alpha_{n}\left(\frac{z f_{n}^{\prime}(z)-f_{n}(z)}{z f_{n}(z)}\right) .
$$

This relation is equivalent to

$$
\frac{F_{n}^{\prime \prime}(z)}{F_{n}^{\prime}(z)}=\alpha_{1}\left(\frac{f_{1}^{\prime}(z)}{f_{1}(z)}-\frac{1}{z}\right)+\cdots+\alpha_{n}\left(\frac{f_{n}^{\prime}(z)}{f_{n}(z)}-\frac{1}{z}\right) .
$$

If we multiply the relation (2.4) with $z$, then we obtain

$$
\frac{z F_{n}^{\prime \prime}(z)}{F_{n}^{\prime}(z)}=\sum_{i=1}^{n} \alpha_{i}\left(\frac{z f_{i}^{\prime}(z)}{f_{i}(z)}-1\right)=\sum_{i=1}^{n} \alpha_{i} \frac{z f_{i}^{\prime}(z)}{f_{i}(z)}-\sum_{i=1}^{n} \alpha_{i}
$$

The relation (2.5) is equivalent to

$$
\frac{z F_{n}^{\prime \prime}(z)}{F_{n}^{\prime}(z)}+1=\sum_{i=1}^{n} \alpha_{i} \frac{z f_{i}^{\prime}(z)}{f_{i}(z)}-\sum_{i=1}^{n} \alpha_{i}+1
$$


This relation is equivalent to

$$
\frac{z F_{n}^{\prime \prime}(z)}{F_{n}^{\prime}(z)}+1=\sum_{i=1}^{n} \alpha_{i}\left(\frac{z f_{i}^{\prime}(z)}{f_{i}(z)}-\beta_{i}\right)+\sum_{i=1}^{n} \alpha_{i} \beta_{i}-\sum_{i=1}^{n} \alpha_{i}+1
$$

We calculate the real part from both terms of the above equality and obtain

$$
\boldsymbol{\operatorname { R e }}\left(\frac{z F_{n}^{\prime \prime}(z)}{F_{n}^{\prime}(z)}+1\right)=\sum_{i=1}^{n} \alpha_{i} \operatorname{Re}\left(\frac{z f_{i}^{\prime}(z)}{f_{i}(z)}-\beta_{i}\right)+\sum_{i=1}^{n} \alpha_{i} \beta_{i}-\sum_{i=1}^{n} \alpha_{i}+1 .
$$

Because $f_{i} \in S_{p}\left(\beta_{i}\right)$ for $i=\{1, \ldots, n\}$, we apply in the above relation inequality (1.3) and obtain

$$
\operatorname{Re}\left(\frac{z F_{n}^{\prime \prime}(z)}{F_{n}^{\prime}(z)}+1\right)>\sum_{i=1}^{n} \alpha_{i}\left|\frac{z f_{i}^{\prime}(z)}{f_{i}(z)}-1\right|+\sum_{i=1}^{n} \alpha_{i}\left(\beta_{i}-1\right)+1 .
$$

Since $\alpha_{i}\left|z f_{i}^{\prime}(z) / f_{i}(z)-1\right|>0$ for all $i \in\{1, \ldots, n\}$, we obtain that

$$
\operatorname{Re}\left(\frac{z F_{n}^{\prime \prime}(z)}{F_{n}^{\prime}(z)}+1\right)>\sum_{i=1}^{n} \alpha_{i}\left(\beta_{i}-1\right)+1
$$

So, $F_{n}$ is convex of order $\sum_{i=1}^{n} \alpha_{i}\left(\beta_{i}-1\right)+1$.

Corollary 2.2. Let $\alpha_{i}, i \in\{1, \ldots, n\}$ be real positive numbers and $f_{i} \in S_{p}(\beta)$ for $i \in\{1, \ldots, n\}$. If

$$
0<\sum_{i=1}^{n} \alpha_{i} \leq \frac{1}{1-\beta^{\prime}}
$$

then the function $F_{n}$ is convex of order $(\beta-1) \sum_{i=1}^{n} \alpha_{i}+1$.

Proof. In Theorem 2.1, we consider $\beta_{1}=\beta_{2}=\cdots=\beta_{n}=\beta$.

Remark 2.3. If $\beta=0$ and $\sum_{i=1}^{n} \alpha_{i}=1$, then

$$
\operatorname{Re}\left(\frac{z F_{n}^{\prime \prime}(z)}{F_{n}^{\prime}(z)}+1\right)>0
$$

so $F_{n}$ is a convex function.

Corollary 2.4. Let $\gamma$ be a real number, $\gamma>0$. Suppose that the functions $f \in S_{p}(\beta)$ and $0<\gamma \leq$ $1 /(1-\beta)$. In these conditions, the function $F_{1}(z)=\int_{0}^{z}(f(t) / t)^{\gamma} d t$ is convex of order $(\beta-1) \gamma+1$.

Proof. In Corollary 2.2, we consider $n=1$.

Corollary 2.5. Let $f \in S_{p}(\beta)$ and consider the integral operator of Alexander, $F(z)=\int_{0}^{z}(f(t) / t) d t$. In this condition, $F$ is convex by the order $\beta$.

Proof. We have

$$
\frac{z F^{\prime \prime}(z)}{F^{\prime}(z)}=\frac{z f^{\prime}(z)}{f(z)}-1
$$


From (2.13), we have

$$
\operatorname{Re}\left(\frac{z F^{\prime \prime}(z)}{F^{\prime}(z)}+1\right)=\operatorname{Re}\left(\frac{z f^{\prime}(z)}{f(z)}-\beta\right)+\beta>\left|\frac{z f^{\prime}(z)}{f(z)}-1\right|+\beta>\beta .
$$

So, the relation (2.14) implies that the Alexander operator is convex.

\section{References}

[1] F. Ronning, "Uniformly convex functions and a corresponding class of starlike functions," Proceedings of the American Mathematical Society, vol. 118, no. 1, pp. 189-196, 1993.

[2] D. Breaz and N. Breaz, "Two integral operators," Studia Universitatis Babeş-Bolyai, Mathematica, vol. 47, no. 3, pp. 13-19, 2002. 